\title{
Argumentation in politischen Texten. Eine textlinguistische Untersuchung anhand von ausgewählter Rede Christian Kerns Mahmoud Sobhy Abou Yousef
}

Assistent an der Abteilung für deutsche Sprache und Literatur Philosophische Fakultät, Universität Menoufia

\begin{abstract}
In der Politik findet man häufig argumentative Texte, in denen man den Hörer/Leser von etwas überzeugen will, was z. B. bei politischen Reden der Fall ist. Die Politiker führen den Menschen bestimmte Gründe, Beweise oder Folgen ihrer Handung an. Ziel ihrer überzeugungsorientierten Argumentation ist es, dass Menschen von bestimmten Sachverhalten zu überzeugen. Sie versuchen also damit, die eigene Einstellung zu stärken und die gegnerische Position zu schwächen. Das Gewicht solcher Texte liegt also in der Regel auf der Argumentation. Die Analyse der Argumentation bzw. der argumentative Themenentfaltung in politischen Reden/Texten steht in diesem Beitrag im Vordergrund. Dabei wird die Struktur und die Funktion der Argumentation anhand von einer Rede des ehemaligen österreichischen Bundeskanzlers und SPÖ-Obmanns Christian Kern untersucht. Die Rede wird als Textsorte mit Methoden der linguistischen Textanalyse ermittelt. Der Beitrag behandelt in diesem Rahmen die folgenden Fragen: Wie wird in der politischen Rede argumentiert? Wie werden die Textthemen argumentativ entwickelt? Wie werden Argumente rekonstruiert und beurteilt? Welche Funktionen haben die Argumentationen? Und anschließend Welcher Zusammenhang besteht zwischen der Textfunktion und der argumentativen Themenentfalutung in politischer Rede?
\end{abstract}

Faculty Of Arts Journal 


\section{Einleitung}

Dr Yaser ATTAR / Dr Mohsen DHIEB,

Die Politik ist wie ein Spiel; die Wörter richtig einzusetzen und den Adressaten gut zu überzeugen sind die Techniken des Spiels, mit denen man entweder siegen oder verlieren kann. In der gegenwärtigen Zeit spielt die Politik eine große Rolle, weil sie die Geschehnisse in der Welt regiert und über die Zukunft des Landes entscheidet. Ihre Wirkung betrifft alle Bereiche des menschlichen Lebens.

Sprache und Politik kreuzen sich täglich. Die Beziehung von Sprache und Politik wird häufig von vielen diskutiert, da die Politiker ihre Worte vorsichtig einsetzen müssen, um eine erfolgreiche Rede zu erzielen. Einige Autoren meinen, dass die Sprache die Politik wirksamer macht: „Alles politische Handeln [ist] sprachliches Handeln" (Holly 1990, S. 8). Politische Sachverhalte werden mit und durch die Sprache interpretiert. Politiker müssen die Bürger informieren, politisches Handeln begründen und Gründe dafür anführen. Sie werten bestimmte Situationen entweder positiv oder negativ. Sie stützen ihre eigene Meinung argumentativ und greifen die gegnerische Meinung argumentativ an. Sie versuchen mit ihren Wörtern den Gegner abzuwerten, die eigenen Anhänger zu mobilisieren und stellen sich glaubwürdig darzustellen.

Der politische Sprachgebrauch hat einige Eigenschaften und Merkmale. Die Wortwahl und die Strategie der Argumentation sind von großer Wichtigkeit für eine wirkungsvolle Rede. „Das Herzstück einer jeden Rede, die das Publikum von einer bestimmten Position überzeugen soll, bildet die Argumentation. Die Überzeugungsabsicht ist für politische Reden der Regelfall“" (Detjen 2014, S. 159). Die Relevanz der Argumentation in den politischen Reden zeigt sich daran, dass immer mehr politische Akteure zu diesem Verfahren neigen, deshalb ist es sehr wichtig, die Argumentation in politischen Texten/Reden gut analysieren zu können.

Der vorliegende Beitrag setzt sich zum Ziel, die Rede des ehemaligen österreichischen Bundeskanzlers und SPÖ-Obmanns 
Applications of GIS in Urban Planning and Transportation

Christian Kern als Textsorte mit Methoden der linguistischen Textanalyse zu ermitteln. Hauptziel der Forschung ist es, die argumentative Themenentfaltung bzw. das argumentative Zusammenspiel der thematischen Strukturebene in politischer Rede Kerns exemplarisch näher $\mathrm{zu}$ beleuchten und die kommunikativ-funktionalen Textmerkmale zu untersuchen. Dabei wird die Struktur und Funktion der Argumentation untersucht.

\section{Was ist Argumentation?}

Die Argumentation als Forschungsgegenstand wurde in unterschiedlichen Bereichen untersucht, wie z. B. in der Logik, der Politik und der Linguistik. In der Politik findet man häufig argumentative Texte, in denen man den Hörer/Leser von etwas überzeugen will, was z. B. bei politischen Reden der Fall ist. Das Gewicht solcher Texte liegt in der Regel auf der Argumentation.

Es ist schon Klar, dass die Zuspitzung der politischen Auseinandersetzungen typisch für das Politische Leben ist. Daher greifen die Politiker in ihrer Reden zum sprachlichen Verfahren der Argumentation, um etwas Strittiges zu klären und damit die anderen von ihrer Positionen zu überzeugen. Man geht also davon aus, dass „am Anfang einer Argumentation eine strittige Aussage [steht]" (Eggler 2006, S.5), die begründet werden soll, was bei politischen Reden der Fall ist. Die meisten Linguisten sind sich darüber einig, dass das Bestehen einer Streitfrage grundlegend für die Argumentation ist. Die Argumentation betrifft aber nicht nur die Strittigkeit, sondern auch die Annahme der Übereinstimmung. Die Strittigkeit wird durch Argumentation bearbeitet, in dem Aussagen implizit oder explizit geäußert werden, von denen die Argumentationspartner bzw. Politiker annehmen, dass ihr Gegner sie aktzeptieret oder akzeptieren sollte. In diesem Sinne beruhen die Begründungen auf Aussagen, 
Dr Yaser ATTAR / Dr Mohsen DHIEB,

die selbst nicht strittig sind, sondern als geltend angenommen werden (vgl. Hannken-Illjes 2018, S. 19f).

Der folgende Abschnitt widmet sich der Argumentation im Allgemeinen. Es wird erklärt, was man unter Argumentation überhaupt versteht und welche Funktionen und Merkmale sie hat. Danach wird die Argumentationsstruktur dargestellt. Anschließend soll auf die Argumentation in Texten näher eingegangen werden.

\subsection{Begriffsbestimmung: Argumentieren, Argumentation und Argument}

Um von Argumentation näher zu sprechen, muss man zwischen den Begriffen Argumentation, Argumentieren und Argument unterscheiden:

Der Begriff „Argumentation“ wird nicht nur in verschiedenen Bereichen verwendet, sondern auch unterschiedlich definiert. Duden (2007, S. 174) definiert die Argumentation als „Darlegung der Argumente, Beweisführung“. Brockhaus Enzyklopädie (1966-1974 zit. nach NE Österreich | Brockhaus 2019) definiert die Argumentation als „Beweisführung mit dem Ziel, die Zustimmung oder den Widerspruch wirklicher oder fiktiver Gesprächspartner zu einer Aussage“. Wörterbuch der deutschen Gegenwartssprache (1964-1977) bezeichnet den Argumentationsbegriff als „Beweisführung, Begründung“.

Nach Lumer (1990, S. 22) wird der Argumentationsbegriff in der Argumentationstheorie in drei verschiedenen Bedeutungen benutzt:

Bedeutung $1 \rightarrow$ Argumentation ist eine geordnete Folge von Aussagen bzw. Urteile mit einem Argumentationsindikator (z. B. Weil, also, da etc.). Die These ist eines dieser Urteile; die anderen sind die Argumente bzw. die Gründe für die These. Der Argumentationsindikator bestimmt die These und die Argumente. 


\section{Applications of GIS in Urban Planning and Transportation}

Bedeutung $2 \rightarrow$ Argumentation ist eine Handlung, in der eine Argumentation 1 vorgetragen wird.

Bedeutung $3 \rightarrow$ Argumentation ist ein Gespräch mehrerer Personen bzw. eine Diskussion mit der Zielsetzung, durch den Gebrach von Argumentationen 2 einen Konsens in einer oder mehreren Streitfragen zu schaffen.

Der Ausdruck "Argumentation" hat alltagssprachlich nur die oben erläuterten Bedeutungen 1 und 2. In dem vorliegenden Beitrag wird nur die Argumentation und das Argumentieren behandelt und zwar abgesehen von ihrer Eingliederung in Diskussionen.

Die Argumentation als Oberbegriff lässt sich am besten von Klaus Bayer (2007, S. 226) wie folgt beschrieben: „sprachliche Handlung einer oder mehrer Personen, bei deren Vollzug ein Argument oder mehrere $\rightarrow$ Argumente geäußert werden, z. B. um Behauptung zu begründen oder Entscheidungen $\mathrm{zu}$ rechtfertigen“.

Bayer definiert also die Argumentation als eine sprachliche Handlung, in der eine These durch Argumente begründet werden soll, was die von Lumer oben erläuterten Bedeutungen 1 und 2 entspricht.

Herrmann/Hoppmann/Stölzgen/Taraman (2012, S. 157) definieren die Argumentation anders, indem er sie als ein Produkt bzw. ein Prozess des Argumentierens betrachtet:

„Sowohl das Produkt des Argumentierens, also die Summe der vorgebrachten Argumente, als auch der Prozess des Argumentierens wird als Argumentation bezeichnet. Die Beziehung einzelner Argumente zueinander in einer Argumentation wird als Argumentationsstruktur bezeichnet".

Diese Definition hebt die von Lumer oben erläuterte Bedeutung 2 von Argumentation hervor, denn nach Öhlschläger kann auch der Ausdruck „Argumentation“ sowohl für die Argumentationhandlungen (bzw. die zu einer Argumentation 1 gehörige Handlung) als auch für die Ergebnisse dieser 
Dr Yaser ATTAR / Dr Mohsen DHIEB,

Handlungen benutzt. Er nennt die Argumentationshandlungen aber „Argumentieren“ (vgl. Öhlschläger 1979, S. 50; und Lumer 1990, S. 22).

Hier wird der Begriff Argumentieren als Prozess hervorgehoben, bei dem laut Hannken-Illjes (2018, S. 21) ,eine Streitfrage durch das Geben und Nehmen von Gründen [bearbeitet wird]“" Argumentieren heißt also Beweise bzw. Gründe anführen. In dieser Hinsicht bezeichnet sie die Argumentation ,als die Abfolge und Kopplung verschiedener Argumente in Bezug auf eine Streitfrage“" (ebd.).

Nachdem nun die Begriffe Argumentation und Argumentieren behandelt werden, soll der Blick von der Argumentation zum Argument gehen: Was ist ein Argument?

Norbert Gratzel (2006, S. 154) definiert der Begriff Argument folgendermaßen:

„Ein Argument in Standardform ist logisch korrekt gdw die Konklusion logisch aus den Prämissen folgt".

Ähnlich wie Grazel betrachtet Bayer (2007, S. 225) das Argument als:

„Folge von Sätzen, bestehend aus mindestens einer Prämisse und genau einer Konklusion. Die Prämissen (Wenn die Sonne scheint, ist es warm. und die Sonne scheint) werden angeführt als Gründe, die Konklusion (Also ist es warm) zu akzeptieren“.

Die Begriffe Argument und Grund werden alltagssprachlich häufig verwechselt, deshalb ist es hier wichtig, zwischen beiden Begriffen zu unterscheiden. Das Argument bezieht sich immer auf eine Streitfrage und enthält Aussagen, die den Übergang vom Grund zur Konklusion rechtfertigen. Ein Grund ist somit eine Aussage innerhalb des Arguments (vgl. Hannken-Illjes 2018, S. 21).

Eine erweiterte Beschreibung des Begriffs Argument führen Herrmann/Hoppmann/Stölzgen/Taraman (2012, S. 157) weiterhin an:

„Ein Argument bindet die Prämissen bzw. Grundannahmen der

Zuhörerschaft an die Behauptung beziehungsweise an den 
Applications of GIS in Urban Planning and Transportation

Standpunkt des Redners an. Im Erfolgsfall wird damit ein Teil der Zustimmung der Zuhörer von ihren Prämissen auf die Behauptung übertragen und damit der Widerstand gegen das rhetorische Ziel reduziert. Die Anbindung der (unstrittigen) Prämissen an die (strittige) Behauptung erfolgt mit Hilfe von Argumentationsschemata bzw. Schlussregeln (z. B. Analogie, Autorität, Kausalität), die zeigen, dass Prämissen und Behauptung in einer Beziehung zueinander stehen".

Man geht davon aus, dass das Argument aus einer Prämisse oder mehrere Prämissen (bzw. Gründe) und genau einer Konklusion (bzw. Behauptung) besteht und damit als die Grundeinheit innerhalb der Argumentation gilt. Die Begriffe Argument und Argumentation können hier aber verwechselt werden, denn der Begriff Argument bezieht sich einmal auf einzelne Prämissen (Argumente), die eine Behauptung begründen soll, und dann wieder auf die Gesamtheit aus Prämissen und Konklusion. Deswegen soll man zwischen den Verwendungsweisen beider Begriffe differenzieren:

In Anlehnung an Georg J. W. Dorn (2006, S. 167f) lassen sich zwei wichtige Verwendungsweisen des Wortes Argument unterscheiden: Im ersten Sprachgebrauch werden mit Argument insbesondere in der logischen Sprachverwendung Abfolgen eng aufeinanderfolgender Äußerungen in Satzform bezeichnet, von denen hier die einen (fast immer die Prämissen des Arguments genannt) für die Rechtigkeit einer anderen (fast immer Konklusion des Arguments genannt) sprechen sollen. Im zweiten Sprachgebrauch wird mit Argument im außerlogischen philosophischen, im argumentationstheoretischen und im alltäglichen Sprachgebrauch solche Äußerungen im Satzform gemeint, die Prämissen in Argumenten gemäß im Sprachgebrauch (1) sind. In dieser argumentationstheoretischen Sprachverwendung heißt das, was sich bei den Logikern Argument nennt, als Argumentation bezeichnet und das, was sich bei den Logikern Konklusion des Arguments nennt, als These der Argumentation. Es besteht also folgende Entsprechung, die dazu 
dient, die Grundbegriffe des Argumentierens deutlich darzulegen:

Logische Sprachverwendung [1] Außerlogische Sprachverwendung [2]

Argument

Prämissen des Arguments

Konklusion des Arguments

$\rightarrow \quad$ Argumentation

$\rightarrow$ Argumente der Argumentation

$\rightarrow \quad$ These der Argumentation

Die oben dargestellte Entsprechung der logischen und außerlogischen bzw. argumentationstheoretischen Sprachverwendungen des Wortes Argument ist durchweg sehr wichtig für das Verstehen der theoretischen Grundlagen der Argumentation. Das Argumentationsverfahren ist weiterhin wie jeder andere Porzess mit Kennzeichen und Funktionen verbunden. Deshalb soll im Folgenden geklärt werden, was eine Argumentation kennzeichnet und warum und wozu man überhaupt argumentiert.

\subsection{Funktionen der Argumentation}

Wenn die politischen Akteure argumentieren, führen sie den Menschen bzw. den Zuhörer bestimmte Gründe, Beweise oder Folgen ihrer Handung an. Ziel ihrer überzeugungsorientierten Argumentation ist es, dass Menschen von bestimmten Maßnahmen überzeugt oder von deren schädlichen Folgen gewarnt werden sollen. Sie versuchen also, die eigene Position zu stärken und die gegnerische Meinung zu schwächen und abzuwerten. Daraus ergibt sich, dass der primäre Zweck des Argumentierens das Überzeugen ist, denn:

„Das Überzeugen verzichtet, jedenfalls in seiner reinen Form, auf emotionale Effekte wie Verheißung und Bedrohung, die Berufung auf Autoritäten und die Eindringlichkeit der retuellen Wiederholung. Und es verzichtet auch auf den Zwang der Gewaltanwendung und -drohung. An deren Stelle tritt hier etwas anderes: die Argumentation" (Weimer 2017, S. 19).

Die Argumentation beruht weiterhin nicht nur auf der Bearbeitung des Dissenses, sondern auch auf der Schaffung von Geltung. Man geht davon aus, dass die Argumentation immer

Faculty Of Arts Journal 
Applications of GIS in Urban Planning and Transportation

über zwei grundlegenden Funktionen verfügt: „Durch Argumentation wird

1) Strittigkeit bearbeitet und

2) Geltung hergestellt“" (Hannken-Illjes 2018, S. 21).

Es gibt aber andere Ansätze, die die Verschärfung einer Streitfrage durchaus auch als Ziel der Argumentation betrachten. So sind zum Beispiel parlamentarische Debatten nicht darauf angelegt, Strittigkeit zu bearbeiten und Konsens zu schaffen, sondern vielmehr darauf, gegensätzliche Einstellungen vor dem Publikum aufzuführen (vgl. ebd., S. 25).

Bayer (2007, S. 64) führt verschiedene Gründe an, die die Argumentation in der Regel als etwas Erstrebenswert erscheinen lassen. Diese Gründe werden hier als die wichtigen Funktionen von Argumentation angesehen:

* Die Argumentation ermöglicht die notwendige Kritik unserer schnellen und irrtums- und verführungsanfälligen Intuition.

* Die Argumente spielen somit für den Aufbau und den Gebrauch unserer Weltbilder eine bedeutende Rolle, besonders bei Erklärungen und bei der für die Erweiterung unseres Wissens entscheidenden Widerlegung von Hypothesen.

* Die argumentative Kritik bietet unbedingt die Möglichkeit, hartnäckige Irrtümer und Vorurteile aufzubrechen.

* Die Argumentative Auseinandersetzung ermöglicht eine relativ friedliche Konfliktlösung und bietet eine Chance, Empathie zu entwickeln und fremde Einstellungen nicht einfach als feindlich und böswillig abzulehnen, sondern ihre Begründung aufzufassen.

Schließlich lässt sich hier sagen, dass die Argumentationen über ihre Erkenntnisfunktion hinaus eine große Zahl weiterer psychischer und sozialer Funktionen haben. Man argumentiert, um die anderen Menschen zu überzeugen; man argumentiert, um die Einstellung seiner eignen Partei oder Interessengruppe zu stärken; man argumentiert, um sich selbst positiv darzustellen und von seinen eigentlichen Zielsetzungen abzulenken (vgl. Bayer 2007, S. 14). 
Dr Yaser ATTAR / Dr Mohsen DHIEB,

Bei der Analyse von Argumentation soll man unbedingt die grundlegende Form der Argumentation bzw. die Komponente der Argumentation ermitteln, auf die im Folgenden näher eingegangen werden soll.

\subsection{Aufbau der Argumentation}

Wie zuvor bereits im Abschnitt 1.2. erwähnt wurde, besteht ein Argument bei den Logikern aus einer Prämisse oder mehreren Prämissen (bzw. Gründe) und genau einer Konklusion (bzw. Behauptung). Die Prämissen sind Aussagen, die als Gründe zur Sicherung und Stützung der Konklusion angeführt werden. In dem Argument

Alle Bürger sind vor dem Gesetz gleichberechtigt $\mathrm{X}$ und $\mathrm{Y}$ sind Bürger

Also $\mathrm{X}$ und $\mathrm{Y}$ sind vor dem Gesetz gleichberechtigt

sind (Alle Bürger sind vor dem Gesetz gleichberechtigt) und (X und $\mathrm{Y}$ sind Bürger) die Prämissen und (Also $\mathrm{X}$ und $\mathrm{Y}$ sind vor dem Gesetz gleichberechtigt) die Konklusion. Diese drei Aussagen bilden im logischen Sinne das Argument, denn:

„[der Begriff Argument] bezieht sich dort manchmal wie in der

Logik auf die Gesamtheit aus Prämissen und Konklusion, manchmal, da im Alltag häufig statt vollständiger Argumente nur einzelne Prämissen genannt werden, nur auf einzelne Prämissen (Bayer 2007, S. 85)“

Im außerlogischen, im argumentationstheoretischen und im alltäglichen Sprachgebrauch wird dieses Argument so formuliert: (X und $\mathrm{Y}$ sind Bürger) sei ein Argument für die These bzw. Behauptung ( $\mathrm{X}$ und $\mathrm{Y}$ sind vor dem Gesetz gleichberechtigt). Weil alle Bürger sind vor dem Gesetz gleichberechtig, sind $\mathrm{X}$ und Y vor dem Gesetz gleichberechtig.

Bei der Analyse der argumentativen Texten soll einfachheitshalber die einzelne Prämissen als Argumente bezeichnet und damit dem oben erläuterten 
Applications of GIS in Urban Planning and Transportation

argumentationstheoretischen und alltäglichen Sprachgebrauch gefolgt werden.

\subsubsection{Das Argumentationsschema von Toulmin}

Bei der Analyse der Argumentation hat St. Toulmin (1985) ein Modell entwickelt, das die allgemeine Argumentationsstruktur darstellt. Es steht nicht den deduktiven und induktiven Argumentformen entgegen. (vgl. Bayer 2007, S. 140). Laut Hannken-Illjes (2018, S. 83) „, [hat sich] das Argumentmodell Toulmins in den letzten sechzig Jahren als das zentrale Modell etabliert". Dieses Modell liegt bis heute den Sprachwissenschaftlen Arbeiten zur Theorie und Analyse der Argumentation zugrunde. Sprachwissenschaftler wie Klaus Bayer (1999/2007²), Josef Kopperschmidt (2000/2014 ${ }^{3}$ ), Marcel Eggler (2006), Kati Hannken-Illjes (2018) u.a. stützen sich bei ihren Überlegungen zur Argumentationstheorie auf Toulmins Ansatz.

Nach Bayer (2007, S. 142) eignet sich das Toulminischen Argumentationsmodell mit den Ahnahmen der Logik. Toulmin unterscheidet hierzu zwischen zwei Varianten von Prämissen, nämlich Übergangsprämissen und Datenprämissen. Die Übergangsprämissen rechtfertigt den Übergang von den Datenargumente zur These bzw. Konklusion bzw. Schlussregel. Sie unterscheiden sich nach ihrer Form und Funktion von den Datenprämissen bzw. Argumente. Toulmin untersucht also, wie Übergangsprämissen und logische Form des Arguments (Schlussregel und Stützung) die Bedeutung der Datenprämissen (Argumente) für die Konklusion (These) begründen, während die Logik die Bedeutung der Gesamtheit der Prämissen für die Konklusion hervorhebt. Das Toulminsschema hat die folgende Form: 


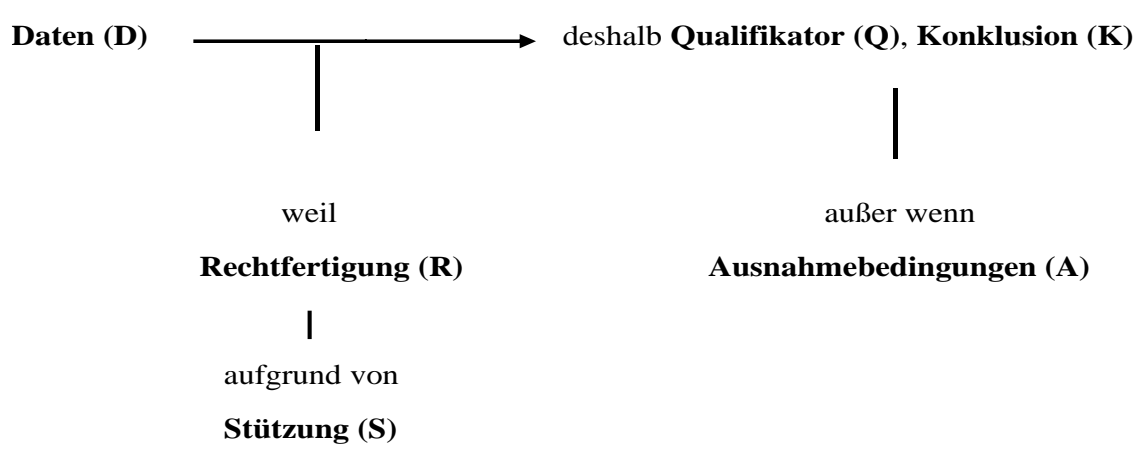

Abb. 1 Argumentationsschema nach Toulmin (zit. nach Bayer 2007, S 140) Mit diesen sechs Bestandteilen legt Toulmin die allgemeine Argumentationsstruktur fest. Durch Argumente (Daten) wird eine These (Konklusion) begründet. Den Übergang von den Daten zur Konklusion rechtfertigt die Schlussregel. Diese Schlussregel ergibt sich aus den Regeln der Deduktion und Induktion. Der Zusammenhang zwischen den Argumenten und der These wird meist durch konditionale Prämissen hergestellt. Die Schlussregel verlangt ihrerseits eine Stützung, denn die theoretische Annahmen benötigen eine Begründung; zudem muss man logische und statistiche Prinzipien herleiten. Die Konklusion kann durch einen Qualifikator (Modaloperator) angezeigt werden, „,er die Stärke des Datums für die Konklusion angibt“ (Hannken-Illjes 2018, S. 84). Es handelt sich hier um den Wahrscheinlichkeitsgrad (z. B. Wahrscheinlich, vermutlich usw.). Eine weitere Kategorie ist die Ausnahmebedingung. Sie benennt die Fälle, in denen die Konklusion gegebenfalls einschränkt gilt (vgl. Bayer 2007, S. 140).

Schließlich ist zu erwähnen, dass Toulmin sein Schema nicht auf ganze Texte angewandt hat, sondern nur auf einzelne Sätze (vgl. Brinker 2018, S. 74). Ausgehend von Toulmins Auffassung, die die Relevanz der Argumentationen in der Alltagssprache hervorhebt, soll im Folgenden die Struktur und Funktion der Argumentation in Reden/Texten behandelt werden, denn:

„Unter Argumentation soll eine Abfolge (Sequenz) von Sprachhandlungen verstanden werden, die zusammen ein oder 
Applications of GIS in Urban Planning and Transportation

weniger komplexes, kohärentes und intentsionales Beziehungsnetz zwischen Aussagen bilden, das der methodischen Einlösung von problematisierten Geltungsansprüchen dient" (Kopperschmidt 2014, S. 59).

\section{Argumentationsstruktur in Texten}

Da der vorliegende Beitrag sich der Argumrntation in politischen Reden aus textlinguistischer Sicht widmt, soll hier die Struktur der Argumentation in Texten klargestellt werden. In diesem Zusammenhang soll zuerst ein Einblick in die Textstrukturanalyse und die wichtigsten Grundformen der thematischen Entfaltung gegeben werden.

Der Text wird in Anlehnung an Brinker (2018., S. 24f) auf zwei eng miteinander verbundenen Ebenen, der grammatischen und der thematischen Ebene untersucht. Auf der grammatischen Strukturebene untersucht man die grammatische Kohärenz, d. h. die für den Textzusammenhang relevanten syntaktischsemantischen Verknüpfungsbeziehungen zwischen aufeinander folgenden Sätzen des Textes Die thematische Beschreibungsebene untersucht hingegen der kognitive Zusammenhang, der zwischen den Sätzen des Textes entsteht, d. h. der thematisch-semantische Zusammenhang eines Textes. Auf dieser Ebene wird vor allem die Struktur des Textinhalts untersucht, die auf den Beziehungen zwischen dem Textthema (Inhaltskern) und den in den propositionalen Komplexen des Textes ausgedrückten Teilinhalten beruht. $\mathrm{Zu}$ den relevanten Analysekategorien dieser Ebene gehören das Textthema und die Form der Themenentfaltung. Das Textthema wird im jeweiligen Text unterschiedlich realisiert. Es handelt sich hierbei um die thematische Entfaltung, d.h. „die gedankliche Ausführung des Themas" (ebd. S.57). Brinker (2018, S. 60) unterscheidet folgende vier Typen der Themenentfaltung:

deskriptive Themenentfaltung

narrative Themenentfaltung 
Dr Yaser ATTAR / Dr Mohsen DHIEB,

explikative Themenentfaltung

argumentative Themenentfaltung

Die deskriptive Themenentfaltung ist von der Art des Themas abhängig. Das Thema bezeichnet also einen einmaligen Vorgang bzw. ein Lebewesen oder einen Gegenstand. Deskriptive Texte sind also „beschreibende Texte oder beschreibende Passagen in Texten“ (Kürschner 2017, S. 247). Die deskriptive Themenentfaltung ist für normative Textsorten wie Nachricht, Bericht und Verträge charakteristisch. Die deskriptive Themenentfaltung kommt häufig in appelativen Texten mit der argumentativen Textentfaltung in Verbindung (vgl. Brinker $u$. a. 2018, S. 60-64).

Bei der narrativen Themenentfaltung wird ein Thema durch abgeschlossenes singuläres Ereignis dargestellt (vgl. ebd., S. 66). Es geht hier also um ein komplexes Thema, allerdings nach dem Prinzip der zeitlichen Abfolge. Die narrative Themenentfaltung ist in Erzählungen jeder Art anzusehen. „Der Grundgestus ist hier also UND, DANN“ (Schlichter 2001). Die narrative Themenentfaltung ist für Alltagserzählungen charakteristisch.

Bei der explikativen Themenentfaltung wird ein Sachverhalt (das zu Erklärende) erklärt, wobei diese Erklärung aus bestimmten anderen Sachverhalten (das Erklärende) logisch abgeleitet wird. Die explikative Themenentfaltung ist für solche Textsorte kennzeichend, die die Erweiterung des Wissens als Ziel haben, wie Lehrbuch, populärwissenschaftlicher oder wissenschaftlicher Text (vgl. Brinker u. a. 2018, S. 69-73). Es ist zu erwähnen, dass diese Texte eine Kombination von explikativen, erklärenden und argumentativen Passagen repräsentieren (vgl. Kunčarová 2011, S.25).

Bei der argumentativer Themenentfaltung werden Behauptungen (Thesen) durch Argumente begründet. Die argumentative Themenentfaltung ist vor allem für appellative Texte charakteristisch. Sie ist auch in normativen Texten (z.B. Gerichtsentscheidungen) und in einigen informativen Texten (z.B. Rezensionen und wissenschaftlichen Abhandlungen) (vgl. 
Applications of GIS in Urban Planning and Transportation

Brinker u.a. 2018, S. 80). Da die politische Textsorten auch Eigenschaften von argumentativen Texten aufweisen, was z. B. bei politischen Reden der Fall ist, wird im folgenden Abschnitt die argumentative Themenentfaltung näher behandelt.

\subsection{Argumentative Themenentfaltung}

Brinker (2018, S. 73ff) beruft sich bei der Analyse von Texten mit argumentativer Themenentfaltung auf das Argumentationsmodell von St. Toulmin, in dem er hervorhebt, dass der Emittent eine Behauptung bzw. These (Claim; Konklusion) durch Argumente (Daten) begründet, die das Textthema repräsentiert. Die These und die Argumente bilden demnoch die Grundlage des argumentativen Textes. Den Übergang von der Argumente zur These sichert eine Schlussregel (Warrant). Sie ist eine allgemeine Hypothetische Aussage, die die Form hat: Wenn D, dann C - oder expliziter: Wenn die Daten $\mathrm{x}, \mathrm{y}, \mathrm{z}$ gegeben sind, dann kann man annhmen, dass $\mathrm{C}$. Sobald der Leser/Hörer diese Argumente und die Schlussregel nicht ausreichend findet, braucht er noch eine Stütze (backing). Schlussregel und Stützung sind demgegenüber in argumentativer Texten häufig nicht ausgedrückt. Sie sind implizite Glieder der Argumentation, die in der Argumentationsanalyse explizit gemacht werden müssen. Ausnahmebedingungen (qualifier) für die Argumente und Modaloperator (z.B. vielleicht, wahrscheinlich usw.) für die These werden auch angegeben.

Da Toulmin, wie bereits schon erwähnt wurde, sein Schema nicht auf ganze Texte angewandt hat, sondern nur auf einzelne Sätze, könnte es nicht so einfach für die Analyse ganzer Texte benutzt werden. Hannken-Illjes (2018, S. 89) kritisiert das ToulminModell, in dem sie darauf hingewiesen hat, dass das Modell durch die Position Ausnahmebedingung einen dialogischen Charakter. Eine Ausnahmebedingung wäre also ein potenzielles Gegenargument. Dennoch bleibt ihr Status ambig; stützt sie 
Dr Yaser ATTAR / Dr Mohsen DHIEB,

auschließlich den Modaloperatoren ab oder ist sie eine echte dialogische Entgegnung. Daraus ergibt sich, dass die Ausnahmebedingung aus textlinguistischer Sicht nicht so wichtig ist. Außerdem können die Qualifikatoren bzw. die Modale Operatoren durch die Formulierung der These angegeben werden. Deshalb werden die Kategorien Ausnahmebedingung und Modaloperator in dem von Toulmin vorgeschlagenen Strukturschema weggelassen. Es bleiben also die vier übrigen erläuterten Kategorien. Zudem stellt Brinker (2018, S. 79) auch fest, dass der Zusammenhang von These, Argumenten, Schlussregel und Stützung der Schlussregel wesentlich für die argumentative Themenentfaltung ist. Er führt über das ToulminModell hinaus noch zwei thematischen Kategorien ein, die er als Einbettung und Wertbasis bezeichnet. Bei seiner exemplarischen Analyse von politischen Kommentaren stellt er fest, dass es in einem argumentativen Text auch Aussagen gibt, die eine These in einen größeren historisch-politischen Zusammenhang einordnen. Diese Textteile versteht er als eine thematische Kategorie und nennt sie Einbettung. Sie situiert die These und Argumente d.h. sie ordnet die These und die Argumente in einen bestimmten Kontext ein. Sie schränkt den Argumentationsbereich eines Textes ein und stützt damit auch in gewisser Hinsicht die Argumentation. Die Wertbasis ist die zugrunde liegende Auffassung der gesamten Argumentation. Sie wird bei den Lesern als vorhanden unterstellt. Sie ist auch im Text häufig nur implizit vorhanden (vgl. ebd., S. 78). Schematisch dargestellt ergibt sich nun die folgende Form: 
Applications of GIS in Urban Planning and Transportation

\begin{tabular}{|c|c|}
\hline \multicolumn{2}{|c|}{ Einbettung } \\
\hline $\begin{array}{c}\text { Argumente (D) } \\
\text { (Daten) }\end{array}$ & $\begin{array}{r}\text { These (K) } \\
\text { (Konklusion) }\end{array}$ \\
& \\
& Schlussregel (SR) \\
\hline & \\
Stuitzung der Schlussregel (S)
\end{tabular}

Abb. 2 Das für die Analyse ganzer Texte verwendetes Toulmin-Modell nach Brinker (2018: 79)

Bei der Analyse von Argumentation in politischen Reden/Texten wird also das oben dargestellte von Brinker modifizierte Argumentationsmodell Toulmins verwendet.

Nun ergibt sich die Frage, woran man ein Argument, das in einer Rede oder in einem Text verwendet wird, erkennt. Ein Argument wird also an seiner Funktion erkannt, denn ein Argument ist dasjenige, was eine These einleuchtend machen und sie begründen soll. Als Leser oder Hörer muss man darauf achten, wovon man überzeugt werden soll - das ist die These, und wodurch oder womit man davon überzeugt werden soll - das ist das Argument. Daher muss man zwischen den beiden Begriffen unterscheiden:

Eine These ist die schlüssige Behauptung eines sprechers - das, was er bei den Lesern beweisen will. Man erkennt die These daran, dass sie sich aus allen Argumenten der Rede als Schlussfolgerung ergibt. Bestimmte Wörter und Ausdrücke wie (daher, deshalb, folglich, also, daraus folgt,dass u.ä..) helfen diesen Zusammenhang aufzufinden. Ein Argument hingegen ist das, was dem Hörer einer Rede (bzw. Leser eines Textes) die Behauptung einsichtig, einleuchtend machen soll. Es begründet also die These. Die Argumente sind der logisch einleuchtende 
Dr Yaser ATTAR / Dr Mohsen DHIEB,

Weg zur These hin. Man erkennt das Argument daran, dass es am Anfang eines Satzes oder Nebensatzes steht, der mit bestimme Indikatoren wie ( weil, denn, da, ja, alle, jeder u.ä) beginnt (vgl. Weimer 2017, S. 30). Darüber hinaus sollen bei der Bestimmung der These und Argumente die Propositionen des Textes etwas kürzer gefasst und die implizite Aussagen explizit gemacht werden.

In einer komplexen Argumentationen können die einzelnen Argumente auf zwei unterschiedliche Weisen zusammenhängen: Sie können untergeordnet oder gleichgeordnet auftreten:

* Untergeordnete Argumente: Dabei handelt es sich hier um Argumente, die sich gegenseitig stützen. Sie begründen und stützen die Ausgangsargument weiter.

Gleiggeordnete Argumente: Dabei stützen die alle angeordneten Argumente den gleichen Standpunkt. Hier werden zur Begründung der Behauptung mehrere Argumente herangezogen, die nebeneinander stehen (vgl. Herrmann u.a. 2012, S. 45f; vgl. Mylonas/Brüning 2013, S. 129).

Nun ist es schon klar, dass die These und die Argumente die Grundlage eines argumentativen Textes bilden. Weitere Kategorien des Toulmin-Modells müssen nicht unbedingt im Text realisiert. Die Schlussregel und die Stützung sind implizite Aussagen, die expilzit gemacht werden sollen. Eine Stützung kann auch im Text direkt ausgedrückt werden.

Bisher erfährt man, welche Komponente die Argumentation in ihrer eignen Struktur hat und woran man sie in einem Text erkennen kann. Aber wie wäre es denn, wenn man es in einem Text mit mehreren Argumentationen zu tun hat? Laut Eggler (2006, S. 77) kann eine argumentative Teilillokution praktisch allen Propositionen eines Textes vom Leser/Hörer entweder direkt oder indirekt via Inferenz zugeordnet werden, was man Superstruktur bezeichnet. Jeder argumentative Schritt auf einer bestimmten These hin weist eine Superstruktur. Makro-Texte unterscheiden sich von Mikro-Texte dadurch, dass sie aus einer hierarchische Aeinanderfügung und Verschachtlungen solcher Superstrukturen bestehen. Die oberste dieser Superstrukturen - 
Applications of GIS in Urban Planning and Transportation

also diejenige, welche die zentrale These, die damit verbundenen Argumente und Schlussregel sowie die Stützung beinhält, wird als Makrostruktur eines Textes bzw. Makropropositionen bezeichnet. Die restlichen Superstrukturen haben den Status von Mirkrostrukturen bzw. Mikropropositionen.

In dieser Hinsicht hat Brinker an konkreten kurzen Textbeispielen die Argumentationsstruktur aufgezeigt. Er stellt dabei fest, dass der Text immer eine Hauptthema bzw. Hauptthese und Nebenthemen bzw. Nebenthesen hat. Die Nebenthema bzw. Nebenthese situiert die Hauptthese und kann damit als Einbettung betrachtet werden. (vgl. Brinker 2018, S.127f).

Die theoretischen Grundlagen der Argumentationstheorie wurden bis hierher durch Beispiele beschrieben. Eine Analyse einzelner Argumentationsstruktur sollte also die Theorie darstellen. Die Argumentionsanalyse als Methode ist dabei noch nicht explizit Gegenstand geworden. Sie ist eine Möglichkeit der Textanalyse, die entsprechend der verschiedenen Perspektiven auf Argumentation, verschiedene Aspekte der Argumentation in den Blick nehmen kann (vgl. Hannken-Illjes 2018, S. 115). Deshalb soll im Folgenden beispielsweise die Argumentationsanalyse bei der Untersuchung umfangreicher Texte bzw. politischer Reden anhand von einer Rede des ehemaligen österreichischen Bundeskanzlers und SPÖ-Bundesparteiobmanns Christian Kern vorgestellt werden.

\subsection{Analyse der Rede von Christian Kern im} Nationalrat am 31.1.2018

- Analyse des Kontextes

Die Rede ist eine dissensorientierte Parlamentarische Debattenrede. Der Redner ist SPÖ-Parteivorsitzender Christian kern. Die Position des Redners als die des Oppositionsführers bestimmt in vielen Hinsichten seine Redeweise. Kern kritisiert 
Dr Yaser ATTAR / Dr Mohsen DHIEB,

die Abschaffung der Aktion 20.000 für Langzeitarbeitslose über 50-jährigen und verteidigte sie im Nationalrat. Er will mit seiner Rede dieses Job-Programm Aktion 20000 beibehalten, das von ihm während seines Kanzleramtes umgesetzt wurde. Diese Rede wurde am Mittwoch, den 31. Jänner 2018 im Nationalrat an den Nationalratpräsidenten, die $\neg$ Abgeordneten des Nationalrates vorgetragen. Sie ist sowohl für die Regierungskoalition besonders die Sozialministerin als auch für das Österreichische Volk bestimmt. Die Rede ist durch das österreichische Fernsehen ORF III live übertragen. Sie wurde von Kern mündlich vorgetragen, dann wurde sie auch auf die Webseite des Österreichischen Parlaments veröffentlicht. Die Rede wird also in verschiedenen Kommunikationsformen realisiert (als Fernsehsendung, als Manuskript bzw. Schrift oder im Internet) und ist dem offiziellen und öffentlichen Handlungsbereich zugeordnet.

- Analyse des Textfunktion

Die Rede verfügt über drei Textfunktionen, nämlich Appellfunktion, Kontaktfunktion und Informationsfunktion. Die Appellfunktion ist aufgrund kontextueller und sprachlicher Indikatoren die dominiernde Funktion in der Rede. Sie wurde in dieser Rede durch expliziten Strukturen und durch weitere grammatische Indikatoren besonders die häufige Verwendung von Imperativsätze realisiert. Die zwei anderen Funktionen, Kontaktfunktion und Informationsfunktion sind in dieser Rede von untergeordneter Bedeutung, denn sie als den Adressatenbezug intensivierende Zusatzfunktionen fungieren.

\section{- Argumentationsanalyse}

Die zu analysierende Rede enthält 71 Segmente, in denen das Thema argumentativ entfaltet wird. Wenn man das von Brinker modifizierte Toulminschema anwendet, kommt man zur folgenden Analyse der Argumentationsstruktur:

Um die Konklusion (Das Job-Programm Aktion 20000 soll beibehalten werden) zu begründen und zu rechtfertigen werden Argumente vom SPÖ-Klubobmann Oppositionsfiihrer Christian 


\section{Applications of GIS in Urban Planning and Transportation}

Kern vorgebracht. Bevor die Argumente dargestellt werden, wird die Argumentation im Text in den Segmenten 1-8 erstens situiert und der Anlass der Rede genannt: Österreich gehe es derzeit wirtschaftlich sehr gut, sagt SPÖ-Obmann Christian Kern Anfang der Rede, neben einem Rekordwachstum und einer positiven Beschäftigungssituation sei auch eine deutliche Sinkung der Staatsschulden feststellbar. Dennoch habe es auch im Jahr 2017 fast eine Million Menschen gegeben, die für einen bestimmten Zeitraum einmal auf Jobsuche waren. Besonders betroffen seien dabei die über 100.000 Langzeitarbeitslosen, die am Arbeitsmarkt oft nur begrenzte Chancen haben. Diese Situierung entspricht also die deskriptive Themenentfaltung und kann zugleich laut Brinker als Einbettung bezeichnet werden.

Nach der Einbettung werden Argumente dargestellt. Die Begründung der These (Das Job-Programm Aktion 20000 soll beibehalten werden). erfolgt durch den Hinweis auf den großen Bedarf an der Aktion 20 000. Zu dieser These gehörendes Argument(1) befindet sich im Segment 13: „Genau für diese Menschen haben wir die Aktion 20000 geplant, Frau Ministerin, weil wir gewusst haben, dass der Markt und die Unternehmen diesen Menschen keine Chance mehr geben, dass diese Auswüchse eines kranken Systems dazu führen, dass Menschen wie Gerd Koppensteiner ausgesondert werden und am Arbeitsmarkt brutal abserviert werden ". Die Schlussregel ist nicht explizit vorhanden aber sie kann folgenderweise formuliert werden: Wenn die Langzeitarbeitslose über 50 Jahren aufgrund ihres Alters ausgesondert werden und am Arbeitsmarkt brutal abserviert werden, dann soll die Regierung ihren Beschluss zur Streichung der für diese Menschen geplanten Aktion 20000 zurücknehmen und sie beibehalten. Die Schlussregel wird hier durch konkreten Beispiele in den Segmenten 9-12 und 23-27 gestuitzt: Um das konkrete Schicksal der Langzeitarbeitslosen über 50 deutlich zu machen, berichtete Kern von zwei 
Dr Yaser ATTAR / Dr Mohsen DHIEB,

Begegnungen mit Menschen, die nur aufgrund ihres Alters nicht mehr den Wiedereinstieg in die Berufswelt schafften.

Weitere Argumente zu der oben erwähnten These können sein: Argument(2) ,(15) Die Aktion 20000 war eines der erfolgreichsten Arbeitsmarktprojekte“. Die Schlussregel ist nicht explizit vorhanden aber sie kann wie folgt ausgedrückt werden: Wenn eine Job-Aktion erfolgreich und bürgerfreundlich ist, dann soll sie beibehalten und nicht von der Regierung gestriechen werden. Gestützt wird die Schlussregel explizit in den Segmenten 16-17 durch die Aussage: ,(17-18) Wir haben sie zunächst einmal in einem Testlauf ausgerollt. Das Ergebnis war, dass in Bezirken, in denen diese Aktion durchgeführt worden ist, das erste Mal seit Jahren die Arbeitslosigkeit bei den Älteren gesunken ist. Das ist ein bemerkenswerter Erfolg “.

Argument(3): „,(19) Wir haben auch gesehen, dass das eine Aktion ist, die in Wirklichkeit gar nicht viel kostet und die wir uns allemal leisten können". Die Schlussregel kann man hier so formulieren: Wenn diese Aktion gar nicht viel kostet und man sie allemal leisten kann, dann soll die Regierung ihren Beschluss zur Streichung dieses Job-Programm zurücknehmen und es beibehalten. Die Schlussregel wird explizit im Segment 20 durch die Aussage gestützt, ,,dass Menschen wie Gerd Koppensteiner, die im Rahmen dieser Aktion beschäftigt sind, 100 Euro pro Monat mehr kosten " (20)

Zu der oben erwähnten These Argument(4) ist impliziert in den Segmenten 21, 37-39 zu finden: Die Aktion 20000 gibt vielen Menschen, die keine Chancen mehr am Arbeitsmarkt haben, ihre Würde und Hoffnung zurück und bietet Perspektiven für die Zukunft. Sie schafft also Arbeitsplätze. Eine Schlussregel ist nur implizit und kann folgendermaßen geäußert werden: Wenn die Aktion 20000 langzeitarbeitslosen über 50 Jahre $20000 \mathrm{Mal}$ Hoffnung, Würde und Chancen bringt und also Arbeitsplätze schafft, dann soll sie beibehalten und nicht von der Regierung gestriechen werden. Eine Stützung ist gar nicht ausgedrückt. Man könnte darauf hindeuten, dass diese Schlussregel einem Faculty Of Arts Journal 
Applications of GIS in Urban Planning and Transportation

anerkannten Bewertungsprinzip der Alltagswelt entspricht. (Wenn ein Job-Programm bürgerfreundlich und schafft Arbeitsplätze, dann ist es positiv zu bewerten).

Kern führte weiter in den Segmenten 47-69 und übte Druck auf die ÖVP-FPÖ-Regierung. Er Kritisierte ihre arbeitsmarktpolitischen Maßnahmen. Er betrachtet diese Maßnahmen als Angriffe auf den Sozialstaat und Arbeitsuchende. Mit seinem Druck will er die Sozialministerin Beate Hartinger-Klein (FPÖ) und die ÖVP-FPÖ-Regierung zwingen, ihren Beschluss zur Streichung der äußerst erfolgreichen Aktion 20000 zurücknehmen. Man geht davon aus, dass es sich hier um ein weiteres Argument(5) handelt. Man kann es folgendermaßen ausformulieren: Die arbeitsmarktpolitischen Maßnahmen der ÖVP-FPÖ-Regierung sind Angriffe auf den Sozialstaat und Arbeitsuchende. Dieses Argument gilt als Argument des inneren Widerspruchs bzw. Spiegelargument, welches polemisch und analytisch Gedankenfehler des Gegners aufdeckt und nutzt dessen Fehler zur Stützung der eignen Einstellung. Kern unterstellt die Absichten des Bundeskanzlers und des Vizekanzlers, Kurz (ÖVP) und Strache (FPÖ) und stellt damit ihre Intentionen bloß, um sie zur Beibehaltung des Jobprogramms Aktion $20000 \mathrm{zu}$ zwingen.

Nachdem SPÖ-Klubobmann Kern die oben erwähnten Argumente vorgebracht hat, drückte er zum Schluss seine Konklusion bzw. Seine These (Die Aktion 20000 soll beibehalten und nicht von der Regierung gestriechen werden) in den Segmenten 70- 71 aus: „, Mein Schlusssatz lautet: Sehr geehrte Frau Sozialministerin, überlegen Sie sich diese Entscheidung noch einmal! Es gibt so viele Betroffene, die diese Aktion brauchen, und meine Bitte und mein Wunsch wären, dass wir losgelöst von parteipolitischer Taktik überlegen, was wir für diese Menschen tun können. - Danke“. 
Die gesamte Argumentation beruht schließlich auf einer bestimmten Auffassung vom Wesen der Politik, worin die arbeitsmarktpolitischen Maßnahmen der Regierung gegen den Sozialstaat und Arbeitsuchende negativ bewertet werden. Die Wertbasis wird in dieser Rede implizit in den Segmenten 21 und 40 zum Ausdruck gebracht. Sie kann folgendermaßen formuliert: Die aktive Arbeitsmarktpolitik soll vor allem Personen unterstützen, die durch verschiedene Hemmnisse erschwert auf eine offene Stelle zu vermitteln sind.

Um die Analyse deutlich darzustellen, sollen die Propositionen des Textes etwas knapper gefasst und die implizite Aussagen explizit gemacht werden, was im Folgenden vorgestellt wird:

\section{These (Konklusion):}

* Die ÖVP-FPÖ-Regierung soll ihren Beschluss zur Streichung der Aktion 20000 zurücknehmen. (Das Job-Programm Aktion 20000 soll beibehalten werden) (Segmente 70-71)

\section{Argumente (Daten):}

1) Die Langzeitarbeitslose über 50 Jahren werden aufgrund ihres Alters ausgesondert werden und am Arbeitsmarkt brutal abserviert. (Segment 13)

Schlussregel:

Wenn die Langzeitarbeitslose über 50 Jahren aufgrund ihres Alters ausgesondert werden und am Arbeitsmarkt brutal abserviert werden, dann soll die Regierung ihren Beschluss zur Streichung der für diese Menschen geplanten Aktion 20000 zurücknehmen und sie beibehalten.

Stützung:

Die Schlussregel wird hier durch konkreten Beispiele gestützt: Um das konkrete Schicksal der Langzeitarbeitslosen über 50 deutlich zu machen, berichtete Kern von zwei Begegnungen mit Menschen, die nur aufgrund ihres Alters nicht mehr den Wiedereinstieg in die Berufswelt schafften. (Segmente 9-12 und 23-27)

2) Die Aktion 20000 war eines der erfolgreichsten Arbeitsmarktprojekte. (Segment 15)

Schlussregel:

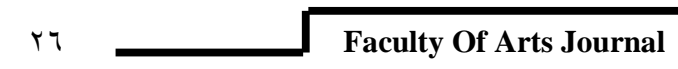




\section{Applications of GIS in Urban Planning and Transportation}

Wenn eine Job-Aktion erfolgreich und bürgerfreundlich ist, dann soll sie beibehalten und nicht von der Regierung gestriechen werden.

\section{Stützung:}

Aufgrund eines Testlaufs wurde festgestellt, dass zum ersten Mal seit Jahren in jenen Bezirken, wo diese Maßnahme umgesetzt wurde, die Arbeitslosenrate bei älteren Menschen gesunken ist. (Segmente 16-17)

3) Die Aktion 20000 kostet gar nicht viel und man sie allemal leisten kann. (Segment 19)

\section{Schlussregel:}

Wenn diese Aktion gar nicht viel kostet und man sie allemal leisten kann, dann soll die Regierung ihren Beschluss zur Streichung dieses Job-Programm zurücknehmen und es beibehalten.

\section{Stützung:}

Menschen wie Gerd Koppensteiner, die im Rahmen dieser Aktion beschäftigt sind, kosten 100 Euro pro Monat mehr. (Segment 20)

4) Die Aktion 20000 gibt vielen Menschen, die keine Chancen mehr am Arbeitsmarkt haben, ihre Würde und Hoffnung zurück und bietet Perspektiven für die Zukunft. Sie schafft also Arbeitsplätze. (impliziert in den Segmenten 21, 37-39)

Schlussregel:

Wenn die Aktion 20000 langzeitarbeitslosen über 50 Jahre 20000 Mal Hoffnung, Würde und Chancen bringt und also Arbeitsplätze schafft, dann soll sie beibehalten und nicht von der Regierung gestriechen werden.

Der gesamte Argumentationszusammenhang kann schematisch folgendermaßen dargestellt werden: 


\begin{tabular}{|l|l|}
\hline \multicolumn{2}{|c|}{ Einbettung (Segmente 1-7) } \\
\hline $\begin{array}{l}\text { Argumente (D) } \\
\text { (Segmente 13,15,19; impliziert in 21, 37-39) }\end{array}$ & $\begin{array}{c}\text { These (K) } \\
\text { (Segment 70) }\end{array}$ \\
\hline Stützung der Schlussregel (Segmente 9-12/23-27, 16-17, 20)
\end{tabular}

\section{Fazit}

- Die politische Rede ist eine von PolitikerInnen emittierte mündliche argumentative Fachtextsorte, die in der Regel über zwei wichtigen Funktionen verfügt, nämlich: eine dominierende Appellfunktion und eine stützende Informationsfunktion.

- Das Gewicht der politischen Rede liegt auf der Argumentation, jedoch werden innerhalb der argumentativen Themenentfaltung deskriptive Passagen eingesetzt, um die Textthese zu stützen. Das deskriptive Prinzip gilt hier als Informationsbasis für die zu begründende These und die Argumente. Sie machen also die Begründung komplex. Man geht davon aus, dass in politischer Rede die Textfunktion, dh. die dominierende Appellfunktion und die stützende Informationsfunktion, im Einklang mit der Entfaltung des Themas, dh. die argumentative Themenentfaltung und die dazugehörigen deskriptiven Passagen, steht.

- Die Textanalyse lässt sich als Gegenstand der Linguistik sehen, welcher sich nun nicht nur mit der traditionallen Analyse von geschriebenen Texten beschäftigt, sondern vielmehr die gesprochene 
Applications of GIS in Urban Planning and Transportation

Sprache behandelt. Bei der Textanalyse kann auch noch einige Analyseschritte erweitert werden, die sich mit der spezifischen Textsorten eignen, wobei in der Regel Ausprägungen von Textanalyse unterschieden werden können, wie z.B. Analyse von argumentativen Texten, Analyse von narrativen Texten etc.

- Bei der Analyse der Argumentation in Texten ist Identifizierung von Argumentationen und Argumenten von großer Wichtigkeit. Die Propositionen des Textes sollen zunächst etwas kürzer gefasst werden. Die implizite Aussagen hat man auch explizit zu machen. Bei der Bestimmung der Textthese bzw. Thesen kann man von den zentralen Gegenständen des Textes bzw. den dominierenden Referenzträger im Text ausgehen. Man muss die These nicht als Aussage erkennen, die man nur behauptet, sondern als Konklusion aus bestimmten Argumenten. Die Reihenfolge der vorgebrachten Argumente im Text soll auch berücksichtigt werden. Für eine genau Identifizierung von Argumenten im Text sind die vorgebrachten Argumentarten zu beschreiben. Zur Bestimmung der Textthese bzw. Thesen und der Argumente im Text helfen manchmal einige sprachliche Indikatoren bzw. Konnektoren und bestimmte Wendungen, aber sie sollen nicht unbedingt im Text vorhanden sein.

- Das Bestehen einer Streitfrage ist grundlegend für die Argumentation in dissensorientierten politischen Reden, was der Fall bei der untersuchten Rede ist.

- Die Einbettung kommt am Anfang der politischen Rede (bzw. des argumentativen Textes) vor. Sie schafft die Informationsgrundlage, ordnet These und Argumente in einen bestimmten politischen Kontext ein und kann damit auch die Argumentation stützen. Diese Situierung entspricht die deskriptive Themenentfaltung und verfügt also über eine informative Textfunktion.

- Die These und Argumente sind der Baustein der Argumentation in der politischen Rede. Weitere Argumentationskomponente sollen nicht unbedingt im argumentativen Text auftreten. Die Schlussregel sind in argumentativen Texten nicht explizit vorhanden. Ihre Funktion ist immer gleich, die Verbindung bzw. Kopplung des Argumentes an die These. Die Stützung der Schlussregel kann im Text aber selten zum Ausdruck gebracht werden. Die Wertbasis, auf der die gesamte Argumentation beruht, wird meist im Text nicht explizit zum Ausdruck gebracht, sondern ist nur implizit vorhanden. Außerdem kann man sie auch vom Text schließen. In politischen 
Dr Yaser ATTAR / Dr Mohsen DHIEB,

Texten beruht die gesamte Argumentation meistens auf bestimmte Auffassungen im Wesen der Politik.

- Die von Toulmin vorgeschlagenen Kategorien „Ausnahmebedingung“ und „Modaloperator“" sind bei der Analyse von politischen Reden/Texten irrelevant. Darauf kann man verzichten, denn es handelt sich um eine von PolitikerInnen emittierte argumentative Rede, wo es keine dialogische Entgegnung gibt. Außerdem spielen die Modale Operatoren keine große Rolle. Sie können durch die Formulierung der These ausgedrückt werden. Deshalb sind beide Kategorien aus textlinguistischer Sicht unerheblich und verzichtbar.

- In den argumentativen Texten können drei unterschiedliche Vorgehensweisen des Argumentierens unterschieden werden: deduktives Vorgehen, induktives Vorgehen und eine Kombination deduktiver und induktiver Vorgehen. In einer deduktiven Argumentation geht der Redner von einer These aus und leitet von ihr seine Argumente ab. In einer induktiven Argumentation hingegen geht der Redner von einem Argument (bzw. Argumenten) aus, das zu seiner These hinführt, was der fall bei der untersuchten Rede ist. Bei der Kombination von deduktiven und induktiven Vorgehen schließlich geht der Redner von einer Behauptung bzw. These aus, leitet von ihr seine Argumente ab und formuliert die These zum Schluss wieder aus.

- Die Funktionen von Argumentation in politischen Reden sind vor allem die Bearbeitung der Strittigkeit und Schaffung der Geltung. Eine Verschärfung der Strittigkeit kann auch als Funktion der Argumentation angesehen werden, indem der Redner gegensätzliche Einstellungen vor dem Publikum vorlegt. Darüber hinaus verfügen die Argumentation in politischen Reden grundsätzlich über psychische und soziale Funktionen. Man argumentiert, um 
Applications of GIS in Urban Planning and Transportation

sich selbst positiv darzustellen und die Position seiner eignen Gruppe zu stärken. Die Funktionen von Argumentation sind mit der Appelfunktion kompatibel, denn der Redner fordert mit der Argumentation die Adressaten auf, seine Meinung zu übernehmen oder eine bestimmte Handlung zu vollziehen. Es handelt sich also um einen argumentativen Appell an die Adressaten.

\section{Literaturverzeichnis}

- Bayer, Klaus (2007): Argument und Argumentation. Logische Grundlagen der Argumentationsanalyse, 2. Auflage. Göttingen: Vadenhoeck \& Ruprecht.

- Brinker, Klaus/Cölfen, Hermann/Pappert, Steffen (2018):

Linguistische Textanalyse. Eine Einführung in Grundbegriffe und Methoden, 9. durchgesehene Auflage. Berlin: Erich Schmidt Verlag.

- Detjen, Joachim (2014): Reden können Demokratie 1. Studien- und Übungsbuch zur politischen Rhetorik. Schwalbach/Ts.: Wochenschau Verlag.

- Dorn, Georg J. W. (2006): Deskriptive Argumente und Argumenthierarchien, in: Kreuzbauer,Günther/ Dorn, Georg (Hg.): Argumentation in Theorie und Praxis. Philosophie und Didaktik des Argumentierens Wien: LIT Verlag Münster.

- Duden - Deutsches universal Wörterbuch (2007), 6. Aufl., Mannheim: Dudenverlag.

- Eggler, Marcel (2006): Argumentationsanalyse textlinguistisch: Argumentative Figuren für und wider den Golfkrieg von 1991. Tübingen: Niemeyer.

- Gratzel, Norbert (2006): Rationale präskriptive Argumente, in: Kreuzbauer,Günther/ Dorn, Georg (Hg.): Argumentation in Theorie und Praxis. Philosophie und Didaktik des Argumentierens Wien: LIT Verlag Münster.

- Hannken-Illjes, Kati (2018): Argumentation. Einführung in die Theorie und Analyse der Argumentation, Tübingen: Narr Francke Attempto Verlag.

- Herrmann, Markus/Hoppmann, Michael/Stölzgen, Karsten/Taraman, Jasmin (2012): Schlüsselkompetenz Argumentation, 2. Aufl., Paderborn: Ferdinand Schöningh. 
- Holly, Werner (1990): Politikersprache. Inszenierungen und Rollenkonflikte im informellen Sprachhandeln eines Bundestagsabgeordneten. 1. Auflage. Berlin : Walter de Gruyter \& Co.

- Kopperschmidt, Josef (2014): Argumentationstheorie. Zur Einführung, 3. Unveränderte Auflage. Hamburg: Junius Verlag.

- Kunčarová, Hana (2011): Die Textsorte Rezension in der Zeitschrift, Diplomarbeit, Die Palacký-Universität Olmütz, URL: https://theses.cz/id/zya4aq/dp_kuncarova.pdf, Abruf am 30.1.2020.

- Kürschner, Wilfried (2017): Grammatisches Kompendium: Systematisches Verzeichnis grammatischer Grundbegriffe, 7. Auflage. Tübingen: Narr Francke Attempto.

- Lumer, Christoph (1990): Praktische Argumentationstheorie: Theoretische Grundlagen, praktische Begründung und Regeln wichtiger Argumentationsarten. Wiesbaden: Vieweg.

- Mylonas, Ioannis / Brüning, Ansgar (2013): Wissenschaftliches Publizieren in der Medizin: Ein Leitfaden. Berlin: Springer Verlag.

- NE Österreich | Brockhaus (2019): Argumentation, URL: http://brockhaus.at/ecs/enzy/article/argumentation, Abruf am 202009-05.

- Öhlschläger, Günther (1979): Linguistische Überlegungen zu einer Theorie der Argumentation. Tübingen: Max Niemeyer Verlag.

- Schlichter, Natalia (2001), Die Thema-Rhema-Struktur in der Textlinguistik, München, GRIN Verlag, URL: https://www.grin.com/document/426, Abruf am 12.02.2020.

- Weimer, Wolfgang (2017): Logisches Argumentieren, Stuttgart: Philipp Reclam.

- Wörterbuch der deutschen Gegenwartssprache (1964-1977), kuratiert und bereitgestellt durch das Digitale Wörterbuch der deutschen Sprache, https://www.dwds.de/d/wb-wdg, Abruf am 05.09.2020.

Die analysierte Rede:

Die Rede des SPÖ-Bundesparteiobmanns Christian Kern im Nationalrat am 31.1.2018, URL: https://www.parlament.gv.at/PAKT/VHG/XXVI/NRSITZ/ NRSITZ_00007/SEITE_0023.html, Abruf am 01.08.2018. (YouTube-Video) unter: https://www.youtube.com/watch?v=NlICpkSjUP0, Abruf am 01.08.2018. 


$$
\text { الحجاج في النصوص السياسية. دراسة من منظور علم لغة النص علي نموذج مختار من }
$$

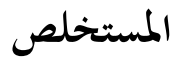

تكثر في لغة السياسة النصوص الحجاجية التى تهدف إلى إقناع المتلقى بما يدور فيها

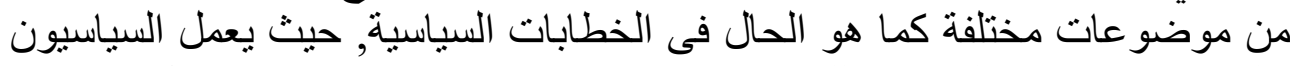

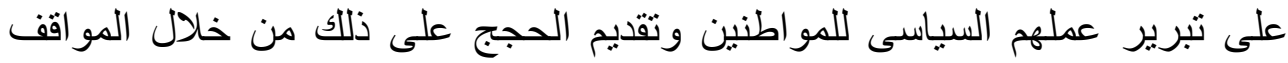

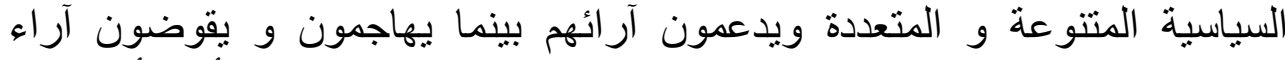

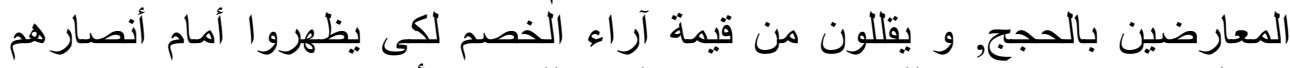

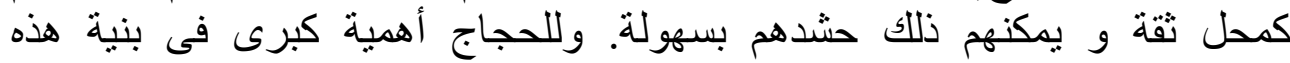

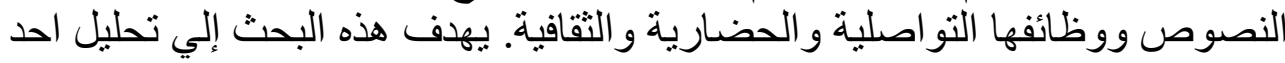

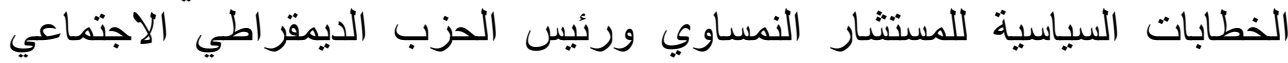

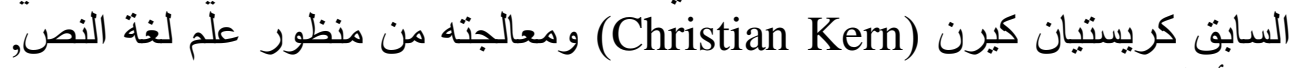

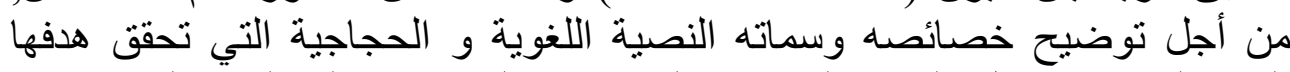

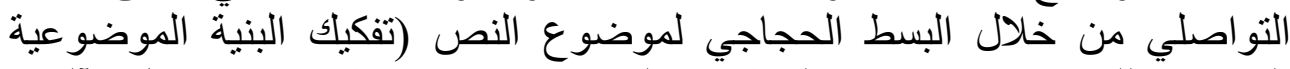

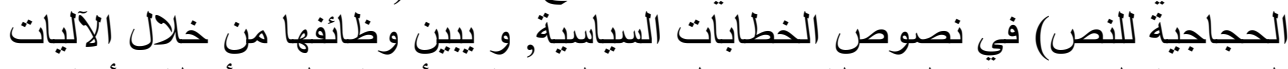

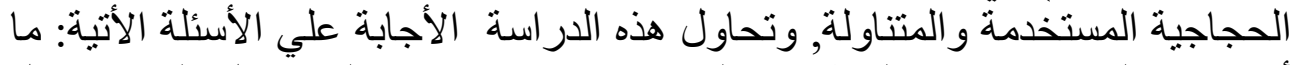

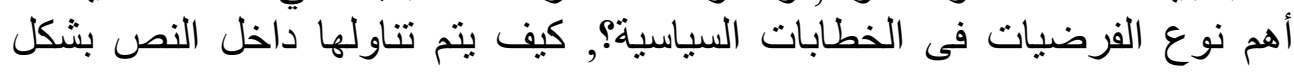

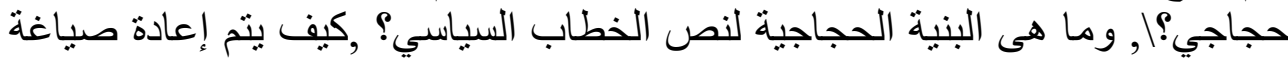

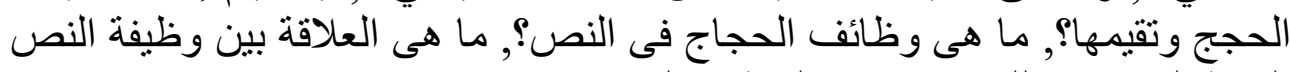
البسط الحجاجي للموضو هئو في الخطاب السياسي؟ 